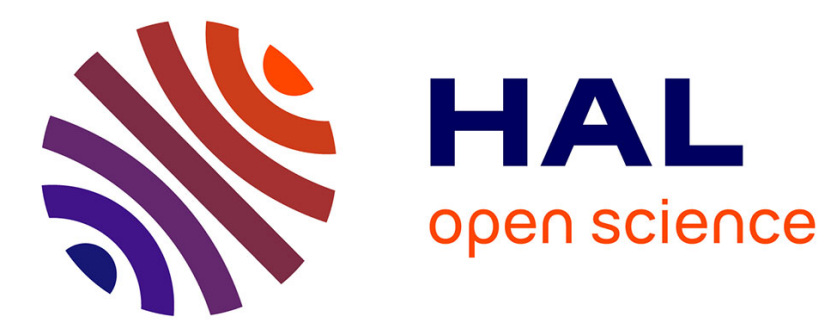

\title{
Design and performance of a miniature free piston expander
}

Sindhu Preetham Burugupally, Leland Weiss

\section{To cite this version:}

Sindhu Preetham Burugupally, Leland Weiss. Design and performance of a miniature free piston expander. Energy, 2019, 170, pp.611-618. 10.1016/j.energy.2018.12.158 . hal-02275711

\section{HAL Id: hal-02275711 \\ https://hal.science/hal-02275711}

Submitted on 1 Sep 2019

HAL is a multi-disciplinary open access archive for the deposit and dissemination of scientific research documents, whether they are published or not. The documents may come from teaching and research institutions in France or abroad, or from public or private research centers.
L'archive ouverte pluridisciplinaire HAL, est destinée au dépôt et à la diffusion de documents scientifiques de niveau recherche, publiés ou non, émanant des établissements d'enseignement et de recherche français ou étrangers, des laboratoires publics ou privés. 


\title{
Design and Performance of a Miniature Free Piston Expander
}

\author{
B. S. Preetham ${ }^{\mathrm{a}, *}$, L. Weiss ${ }^{\mathrm{b}, * *}$ \\ ${ }^{a}$ Department of Mechanical Engineering, Wichita State University, Wichita, KS 67260 USA \\ ${ }^{b}$ Department of Mechanical Engineering, Louisiana Tech University, Ruston, LA 71272 USA
}

\begin{abstract}
The availability of free thermal energy provide an opportunity for power generation at miniature length scales (millimeter). Though free piston based expanders at macro length scales (centimeter and above) are found to be suitable for heat energy harvesting, their implementation at miniature scales are plagued by significant parasitic losses, yielding low thermal efficiencies. Through physics-based models we investigate the behavior and performance of a miniature free piston expander that operates on an open cycle. Here, we explore the design space of the free piston expander with an objective to achieve an efficiency of at least 15\%. Three observations are reported that contribute to higher efficiency operation: (1) a higher injection pressure; (2) an optimum nondimensionalized duration of injection time of 1.5; and (3) softer springs, lower loads, and heavier pistons. A sample calculation showed that a centimeter-sized expander can generate an output power of $2.24 W$ at 18\% efficiency. We observe that both the performance parameters of the expander, namely efficiency and output power are sensitive to injection pressure of the working fluid compared to the time duration of the working fluid injection. Our study reveals that the miniature free piston expander is promising for low temperature waste-heat harvesting.
\end{abstract}

Keywords: Free piston, external combustion, mathematical modeling, open cycle, phase change.

\section{Introduction}

Power generation via miniature thermal energy harvesters, $O\left(10^{-2}\right) \mathrm{m}$, has been a topic of interest due to many foreseeable applications such as environmental and structural health monitoring which require a sustained, reliable milli-Watt power source [1]. An example thermal harvester is a phase-change based harvester which offer the advantage of energy source flexibility, that is, it can operate from different external heat sources. A typical phase-change based harvester setup comprises of four components:

\footnotetext{
${ }^{*}$ Corresponding author

${ }^{* *}$ Corresponding author Email addresses:

sindhupreetham. burugupally@wichita.edu (B. S.

Preetham), lweiss@latech.edu (L. Weiss)
}

a boiler to scavenge available energy and generate pressurized steam, an expander to convert heat energy of the pressurized steam into mechanical work, a condenser to change the state of expended steam into liquid, and a pump to circulate the condensed liquid into the boiler. Typically, low temperature harvesters are based on an Organic Rankine Cycle (ORC) [2, 3, 4, 5] which employs refrigerant (e.g. HCFC-123) as a working fluid [6, 7, 8] in traditional high-speed turbines [9] and crankshaft-based piston expanders [10], and less popular screw [11] and scroll expanders [12]. Most ORC-based harvesters are macroscale systems which benefit from scaling laws that offer high manufacturing tolerances, less heat loss, and less pumping work. Unfortunately, as the size of these thermal systems decrease, the ratio of friction, leakage, and heat losses to input energy be- 
come significant in the expander units, resulting in poor thermal efficiencies [13. A method to improve thermal efficiency is by eliminating the crankshaft assembly and associated friction losses; this can be accomplished by implementing a free-piston architecture of the expander which also offer other advantages - simpler construction and lower fabrication costs.

Most prior research has focused on the design of macroscale free-piston expanders (FPE) [14, 15, 16]. Here, researchers found that FPEs are suitable for heat energy harvesting application. The FPE implementation at miniature scales are not completely studied; however, theoretically, FPE utilization at miniature length scales is challenged by significant parasitic losses such as heat loss and pumping loss, as aforementioned. On the contrary, the scaling laws benefit FPEs in terms of specific output power [13. To evaluate the suitability of FPE at miniature scales and to optimize FPEs, one needs to identify their important design and operating parameters. Prior efforts recorded in literature have made progress on design and operation of thermal harvesters on several fronts - different working fluids (steam, helium, and air) have been considered [14, 15, 17, 18, -different boiler and superheater designs for miniature length scales have been proposed [19, 20] - new lumped parameter-based and numerical-based models of the free piston expander (FPE) have been developed [16, 21]. Many efforts are specific to low temperature waste-heat harvesting and multiple have resulted in real prototypes across different length scales [22, 23, 14, 15, 16, 18,. More recent prototype development has included low grade energy use in ORCstyle free piston expanders with real devices characterized by several groups [14, 15, 16. In these works, a linear electric generator was also incorporated for real-world demonstration of power output. These efforts have produced devices with an output power of up to $96 \mathrm{~W}$ [15] and bore diameters up to $80 \mathrm{~mm}$ [16].

Our present work builds on this growing interest through exploration of free piston expander design space on the smaller scale, specifically in an ORCstyle operation. There is significant opportunity to identify physical and operating parameters in this development space. This includes dynamics and thermodynamic modeling framework that provides in-

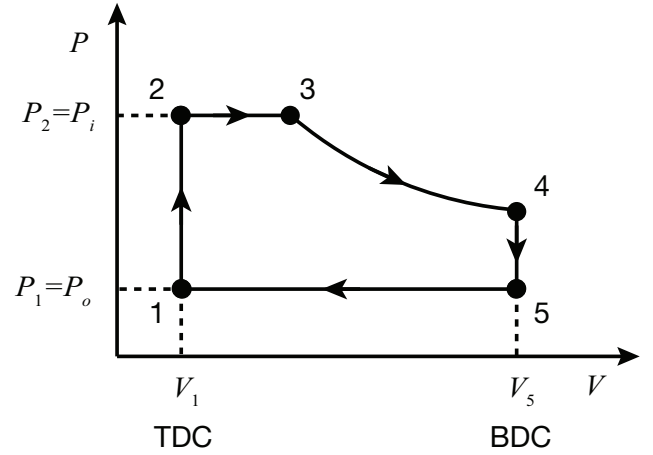

Figure 1: Ideal pressure-volume diagram of the FPE in an open cycle operation. Processes $1 \rightarrow 2$ : First phase of working fluid injection (pressure driven - pressure difference across the valve); $2 \rightarrow 3$ : Second phase of working fluid injection (timed injection for duration $t_{23}$ ); $3 \rightarrow 4$ : Expansion; $4 \rightarrow 5$ : Blowdown phase of exhaust; and $5 \rightarrow 1$ : Displacement phase of exhaust. Note the piston positions at states 1 and 5 are 'Top Dead Center' (TDC) and 'Bottom Dead Center' (BDC), respectively.

sight into the critical parameters and means to optimize FPE performance. This work builds on our own prior, physics-based model 21. Like other works 24, the initial effort modeled the FPE as a closed cycle operation, where the steam injection and exhaust processes were modeled as heat addition and rejection processes respectively. In this work, we model the FPE close to its final real-world operation, that is as an open cycle more similar to the larger scale prototype expanders (Fig. 1).

This work specifically investigates the behavior, performance, and explores the design space of a centimeter-sized FPE modeled to operate on an open cycle (Fig. 1). In this cycle, a hot, pressurized working fluid (air) is injected into the FPE cavity through a valve to produce PdV work at the piston end (Fig. 2. The model is based on a lumped parameter approach developed using first principles. The following sections describe the FPE design, a physics-based model of the FPE, and results that describe FPE behavior. Results show that up to $15 \%$ efficiency can be achieved when supplied with hot, compressed air at $373 \mathrm{~K}$ temperature and $250 \mathrm{kPa}$ pressure. 


\section{FPE design}

The FPE design is similar to a traditional steamexpander system, where hot, pressurized working fluid (steam or vapor) is injected into the FPE cavity ' $\mathrm{CV}$ ' through a valve to produce PdV work by the linear motion $x(t)$ of the piston $m$ (Fig. 2).

The FPE operates on an open cycle comprised of injection $(1 \rightarrow 2 \rightarrow 3)$, expansion $(3 \rightarrow 4)$, and exhaust $(4 \rightarrow 5 \rightarrow 1)$ processes as depicted in Fig. 1. The cycle begins with the piston at state 1 'Top Dead Center' (TDC) where hot, high pressure working fluid is injected into the CV by the opening of the valve. This results in pressurization of the CV and moving of the piston towards 'Bottom Dead Center' (BDC). The valve remains open until the piston reaches state 3 . Note that the first phase of injection process $1 \rightarrow 2$ is pressure driven - pressure difference across the valve, while the second phase of the injection process $2 \rightarrow 3$ is a timed injection for a predetermined time duration $t_{23}$. Then, the valve closes, the expansion process $3 \rightarrow 4$ begins where the CV expands until state 4 . Next, the valve opens and blowdown phase of the exhaust process begins where the expended working fluid is discharged out of the $\mathrm{CV}$ instantaneously and adiabatically to ambient pressure $P_{o}(4 \rightarrow 5)$. Finally, the displacement phase of the exhaust process begins $(5 \rightarrow 1)$ where the piston travels from BDC to TDC scavenging the remaining working fluid from the $\mathrm{CV}$. This completes one operating cycle of the FPE. Note the injection (IR) and expansion (ER) ratios are defined as $\mathrm{IR}=\frac{V_{3}}{V_{1}}$ and $\mathrm{ER}=\frac{V_{4}}{V_{3}}$.

It is worth noting that, unlike standard reciprocating engines where TDC and BDC are geometrically determined, the TDC and BDC in the FPE are determined by the operating conditions.

\section{Model}

A physics-based model of the FPE is developed using the lumped-parameter approach to investigate the FPE behavior, performance, and explore the design space. The FPE is modeled as spring-massdamper system with a hollow cavity CV to contain the working fluid. The injection of working fluid

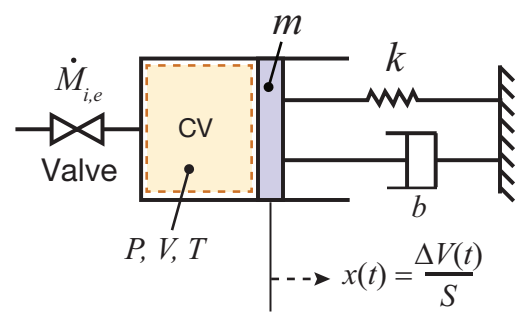

Figure 2: Schematic sketch of the FPE. CV denotes the cavity or control volume with state parameters: pressure $P$, volume $V$, and temperature $T$ at time $t$. The valve on the left is used for the injection or exhaust of the working fluid. The valve is connected to the high pressure working fluid line $\left(P_{i}, T_{i}\right)$ during the injection process, and to the ambient atmosphere $\left(P_{o}, T_{o}\right)$ during the exhaust process.

into the $\mathrm{CV}$ at temperature $T_{i}$ and pressure $P_{i}$ results in the horizontal displacement $x(t)$ of the piston (mass) $m$. The spring with stiffness $k$ helps restore this horizontal position $x(t)$ of the piston $m$. This simulates one of several return-stroke mechanisms in an FPE. Another mechanism is the use of a double-acting FPE where two pistons are mounted to the same piston shaft, both pointed away from each other. The expansion of one piston naturally resets the other. Both mechanisms have historical application in internal combustion configuration [25, 26].

When the displacement of the piston $m$ is zero, the cavity $(\mathrm{CV})$ volume $V_{o}$ is $V_{o}=L S$, where $L$ is the nominal cavity length and $S$ is the cross-sectional area of the cavity. The movement of the piston $m$ is impeded by the damper $b$, which models energy conversion that is the sum of both friction work and useful work. For a steady state operation, the working fluid temperature, $T_{o}+\Delta T(t)$, pressure, $P_{o}+\Delta P(t)$, and cavity volume, $V_{o}+\Delta V(t)$ undergo cyclic variation, where the nominal or ambient and time-varying cyclic components are denoted with the 'o' subscript and $\Delta$, respectively.

The lumped parameter model of the FPE shown in Fig. 2 is derived by applying Newton's second law for the piston $m$, conservation of mass, conservation of energy, an ideal gas model to the cavity CV containing the working fluid, and a linear mass flow-rate equation for the valve. Mathematical statements of these principles are: 


$$
\begin{gathered}
\frac{m}{S^{2}} \Delta \ddot{V}+\frac{b}{S^{2}} \Delta \dot{V}+\frac{k}{S^{2}} \Delta V=\Delta P \\
\dot{M}=\dot{M}_{i}-\dot{M}_{e} \\
h \Delta T+\frac{\mathrm{d}\left(M c_{v} T\right)}{\mathrm{d} t}+P \Delta \dot{V}=\dot{M}_{i} c_{p} T_{i}-\dot{M}_{e} c_{p} T \\
P V=M R T
\end{gathered}
$$$$
\dot{M}_{i, e}= \begin{cases}\dot{M}_{i}=\beta\left(P_{i}-P\right) ; \dot{M}_{e}=0, & \text { process } 1 \rightarrow 3 \\ \dot{M}_{i}=0 ; \dot{M}_{e}=0, & \text { process } 3 \rightarrow 4 \\ \dot{M}_{i}=0 ; \dot{M}_{e}=\lim _{t_{45} \rightarrow 0} \frac{\left(M_{4}-M_{5}\right)}{t_{45}}, & \text { process } 4 \rightarrow 5 \\ \dot{M}_{i}=0 ; \dot{M}_{e}=\beta\left(P-P_{o}\right) & \text { process } 5 \rightarrow 1\end{cases}
$$

where $M$ is the mass of the working fluid in the CV at any given time $t, h$ is a coefficient that models conduction/convection heat losses from the CV to the surroundings, $R$ is the mass-specific gas constant of working fluid, $\beta$ is the mass flow rate coefficient, and $c_{v}$ and $c_{p}$ are the constant volume and constant pressure heat capacities of the working fluid, respectively and are assumed constant over the temperature range. The overdot denotes time derivative, while the subscripts ' $i$ ' and ' $e$ ' denote injection and exhaust processes, respectively.

If the working fluid injection pressure $P_{i}$, temperature $T_{i}$, time duration $t_{23}$, and initial conditions $\Delta V(0), \Delta P(0)$, and $\Delta T(0)$ are specified, Eqs. 15 constitute a nonlinear model for the determination of the intermediate thermodynamic state variables: $\Delta V(t), \Delta P(t)$, and $\Delta T(t)$. As the injection process is periodic, it is assumed that the dependent variables $\Delta V(t), \Delta P(t)$, and $\Delta T(t)$ will also be periodic at steady state.

The nonlinear model, Eqs. 1 5 is first nondimensionalized using the scales in Eq. 6

$$
\Delta \bar{V}=\frac{\Delta V}{V_{o}}, \Delta \bar{P}=\frac{\Delta P}{P_{o}}, \Delta \bar{T}=\frac{\Delta T}{T_{o}}, \text { and } \bar{t}=t \omega
$$

where $\omega=\sqrt{\frac{\gamma P_{o} S^{2}}{m V_{o}}}$ is a reference frequency and the overbar indicates a nondimensional independent or dependent variable. Thus obtained nondimensionalized equations are written in state space format as shown below:

$$
\frac{d \Delta \bar{V}}{d \bar{t}}=\Delta \dot{\bar{V}}
$$

$$
\frac{d \Delta \dot{\bar{V}}}{d \bar{t}}=\frac{\Delta \bar{P}}{\gamma}-\frac{b}{m \omega} \Delta \dot{\bar{V}}-\frac{k}{m \omega^{2}} \Delta \bar{V}
$$

$$
\Delta \dot{\bar{T}}=\frac{1}{M c_{v} T_{o} \omega}\left[\dot{M}_{i} T_{o}\left[R+c_{p} \Delta \bar{T}_{i}-c_{v} \Delta \bar{T}\right]-h T_{o} \Delta \bar{T}\right.
$$

$$
\left.-\dot{M}_{e} R T_{o}(1+\Delta \bar{T}) \quad-P_{o} V_{o} \omega(1+\Delta \bar{P}) \Delta \dot{\bar{V}}\right]
$$

$$
\begin{aligned}
\Delta \dot{\bar{P}}= & \frac{1}{P_{o} V_{o} \omega(1+\Delta \bar{V})}\left[\left(\dot{M}_{i}-\dot{M}_{e}\right) R T_{o}(1+\Delta \bar{T})\right. \\
& \left.+M R T_{o} \omega \Delta \dot{\bar{T}}-P_{o} V_{o} \omega(1+\Delta \bar{P}) \Delta \dot{\bar{V}}\right]
\end{aligned}
$$

Here, it is noteworthy to mention that Eq. 10 is obtained by taking a first derivative of ideal gas law Eq. 4 with respect to the nondimensional time $\bar{t}$.

To generate a pressure-volume diagram, the model Eqs. $7 \sqrt{10}$ is numerically integrated in the order $1 \rightarrow 2 \rightarrow 3 \rightarrow 4 \rightarrow 5 \rightarrow 1$ (Fig. 1) starting with state 1 : $\Delta \bar{V}_{1}, \Delta \bar{P}_{1}, \Delta \bar{T}_{1}, \Delta \dot{\bar{V}}_{1}=0$. A proper choice of state variables at state $1: \Delta \bar{V}_{1}, \Delta \bar{P}_{1}, \Delta \bar{T}_{1}$ are necessary to obtain a steady state solution (see Appendix for details). The resulting volume $\Delta \bar{V}_{2}$, pressure $\Delta \bar{P}_{2}$, and temperature $\Delta \bar{T}_{2}$ from the first phase of the injection process, $1 \rightarrow 2$ is computed by numerically integrating Eqs. 770 from the initial condition $\Delta \bar{V}_{1}, \Delta \bar{P}_{1}, \Delta \bar{T}_{1}, \Delta \bar{V}_{1}=0$ for a time $\bar{t}_{12}$ such that the $\mathrm{CV}$ pressure $P_{2}$ equals injection pressure $P_{2}=P_{i}$. The resulting volume $\Delta \bar{V}_{3}$, pressure $\Delta \bar{P}_{3}$, and temperature $\Delta \bar{T}_{3}$ during the second phase (timed injection) of the injection process, $2 \rightarrow 3$ is computed by numerically integrating Eqs. 770 from the initial condition $\Delta \bar{V}_{2}, \Delta \bar{P}_{2}, \Delta \bar{T}_{2}, \Delta \dot{\bar{V}}_{2}$ for a predetermined 
time duration $\bar{t}_{23}$. The volumes, pressures, and temperatures $\Delta \bar{V}_{4}, \Delta \bar{P}_{4}, \Delta \bar{T}_{4}$ and $\Delta \bar{V}_{1}, \Delta \bar{P}_{1}, \Delta \bar{T}_{1}$ from the expansion and exhaust (displacement phase) processes $3 \rightarrow 4$ and $5 \rightarrow 1$ are computed by numerically integrating Eqs. 7, 10 from the initial conditions $\Delta \bar{V}_{3}, \Delta \bar{P}_{3}, \Delta \bar{T}_{3}, \Delta \bar{V}_{3}$ and $\Delta \bar{V}_{5}, \Delta \bar{P}_{5}, \Delta \bar{T}_{5}, \Delta \dot{\bar{V}}_{5}=0$ for times $\bar{t}_{34}$ and $\bar{t}_{51}$, respectively such that the piston velocity $\Delta \dot{\bar{V}}_{4}=\Delta \dot{\bar{V}}_{1}=0$. It is worth nothing that the times $\bar{t}_{12}, \bar{t}_{34}, \bar{t}_{51}$ are 'a priori' and is determined during the integration. It is assumed that the blowdown process $4 \rightarrow 5$ is instantaneous, isentropic, and occurs at zero piston velocity $\left(\Delta \dot{\bar{V}}_{4}=\Delta \dot{\bar{V}}_{5}=0\right)$ and ambient pressure such that $\Delta \bar{P}_{5}=0$. Therefore, the temperature of the working fluid at the end of the exhaust (blowdown phase) process $4 \rightarrow 5, \Delta \bar{T}_{5}$ is computed using the adiabatic relation given by Eq. 11.

$$
\Delta \bar{T}_{5}=\left(1+\Delta \bar{T}_{4}\right)\left(\frac{1+\Delta \bar{P}_{4}}{1+\Delta \bar{P}_{5}}\right)^{\frac{1-\gamma}{\gamma}}-1
$$

\section{Results and Discussion}

The centimeter-sized FPE shown in Fig. 2 modeled with $m=0.034 \mathrm{~kg}, k=1000 \mathrm{~N} / \mathrm{m}, b=10 \mathrm{~N}-\mathrm{s} / \mathrm{m}, h=0$ $\mathrm{W} / \mathrm{K}, \beta=0.0064 \mathrm{~kg} / \mathrm{Pa}-\mathrm{s}$ and $V_{o}=0.785 \mathrm{~cm}^{3}(1 \mathrm{~cm}$ in diameter and $1 \mathrm{~cm}$ of nominal length) is treated as the reference case. The numerical values of some of these FPE parameters are based on the prior work by the authors [18, 27.

Standard temperature and pressure conditions of $T_{o}=298 \mathrm{~K}$ and $P_{o}=101 \mathrm{kPa}$ are chosen for the ambient state. The working fluid is a hot compressed air, assumed to behave like an ideal gas with specific heat ratio $\gamma=1.4, R=287 \mathrm{~J} / \mathrm{kg}-\mathrm{K}$, and $c_{v}=717 \mathrm{~J} / \mathrm{kg}-\mathrm{K}$. This allows formal concentration on the FPE operating cycle. Future efforts and experiment will consider phase change effects of working fluids already under investigation through experimental boiler development [19]. These are generally 'dry' fluids with a negative slope to the saturation curve. This allows expansion of the fluid within the FPE while maintaining a superheat condition.
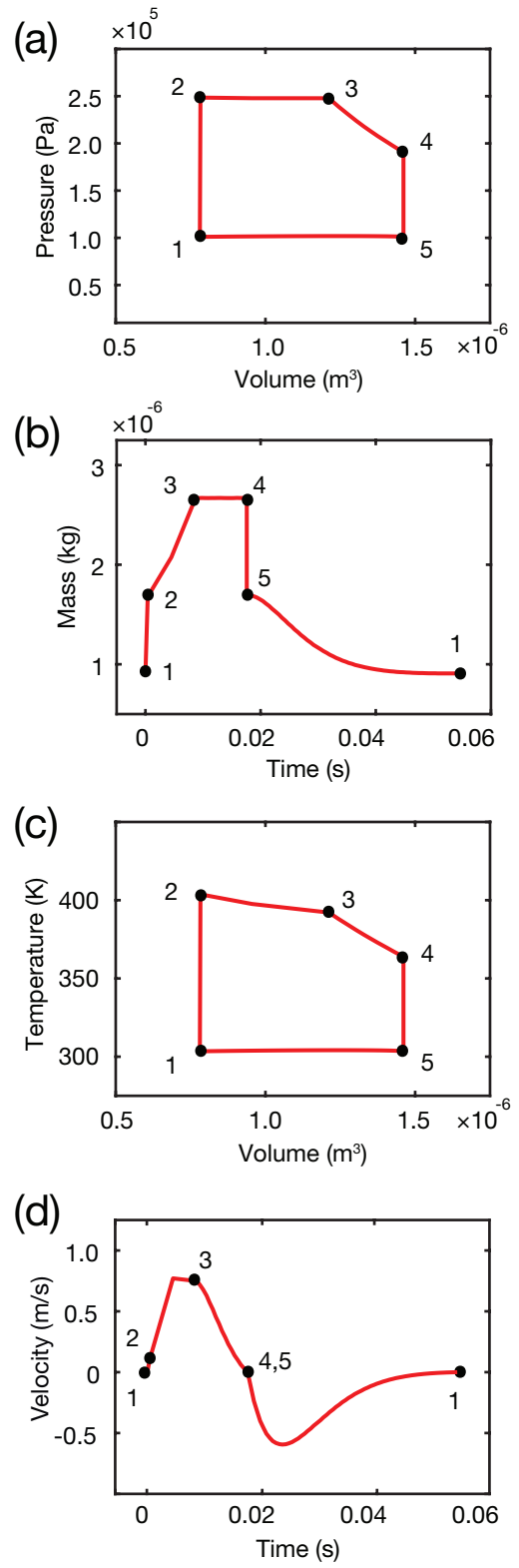

Figure 3: FPE behavior for the reference case with the working fluid injection pressure $P_{i}=250 \mathrm{kPa}$, temperature $T_{i}=373 \mathrm{~K}$, and time duration $\bar{t}_{23}=1.5$. (a) Pressure-Volume (b) Working fluid mass-Time (c) Temperature-Volume (d) Velocity-Time diagrams for one operating cycle. 

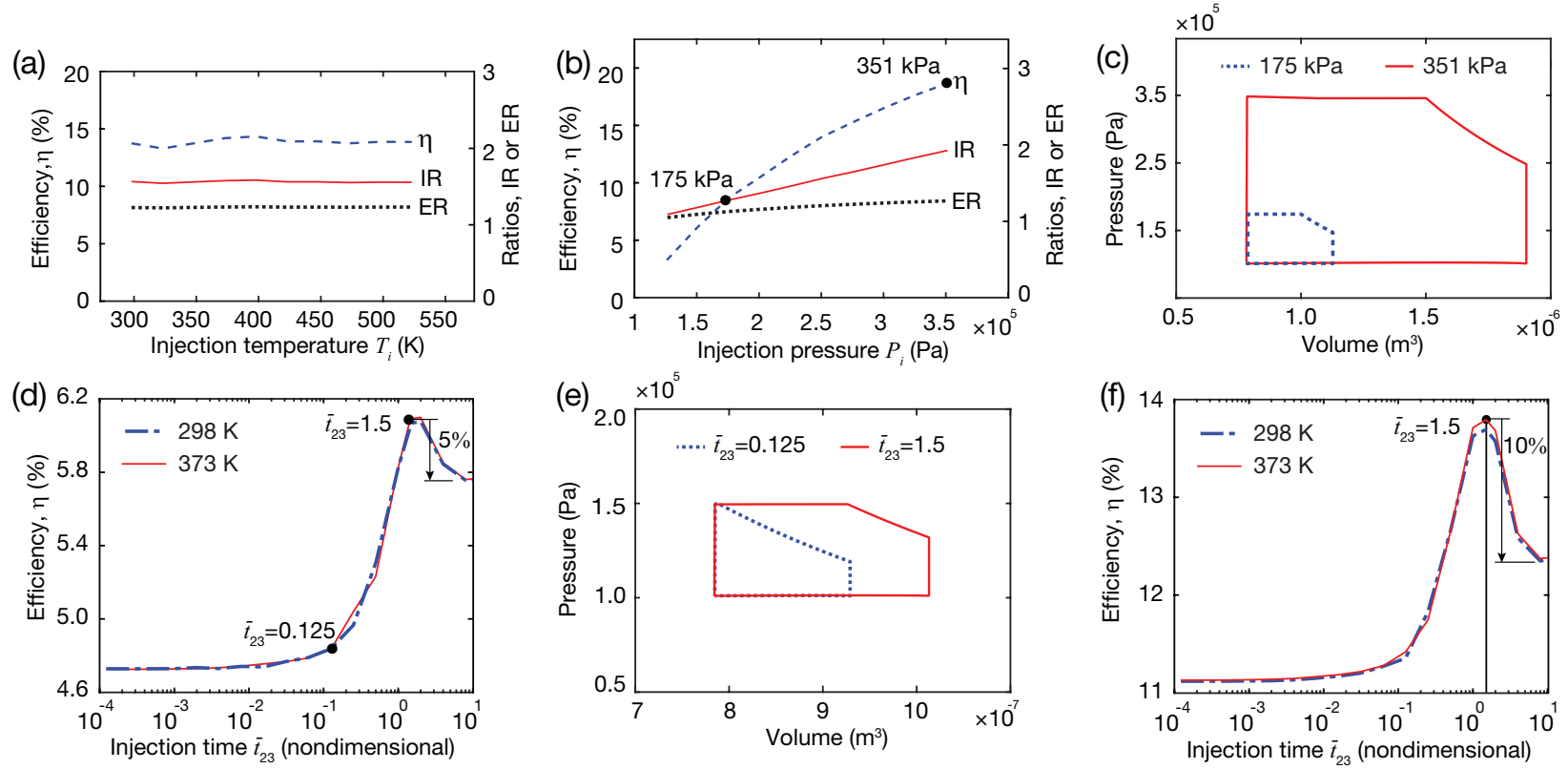

Figure 4: Effect of operating conditions: injection temperature $T_{i}$, injection pressure $P_{i}$, and injection time duration $\bar{t}_{23}$ on FPE efficiency for the reference case. (a) Fixed $P_{i}=250 \mathrm{kPa}$ and $\bar{t}_{23}=1.5$ at different $T_{i} \in[298,523] \mathrm{K}$. (b) Fixed $T_{i}=373$ $\mathrm{K}$ and $\bar{t}_{23}=1.5$ at different $P_{i} \in[126,351] \mathrm{kPa}$. (c) Pressure-Volume diagrams for $P_{i}=175$ and $351 \mathrm{kPa}$ corresponding to sub-figure (b). (d) Fixed $P_{i}=150 \mathrm{kPa}$ and $T_{i}=298,373 \mathrm{~K}$ at different $\bar{t}_{23}$. (e) Pressure-Volume diagrams for $\bar{t}_{23}=0.125$ and 1.5 corresponding to sub-figure (d). (f) Fixed $P_{i}=250 \mathrm{kPa}$ and $T_{i}=298,373 \mathrm{~K}$ at different $\bar{t}_{23}$. Note that the efficiency depends on IR and ER.

\subsection{FPE behavior and performance}

A representative behavior and performance of the FPE with the working fluid injection temperature $T_{i}=373 \mathrm{~K}$, pressure $P_{i}=250 \mathrm{kPa}$, and duration $\bar{t}_{23}=1.5$ for the reference case is shown in Fig. 3 . The pressure-volume diagram for one operating cycle $1 \rightarrow 2 \rightarrow 3 \rightarrow 4 \rightarrow 5 \rightarrow 1$ shows that the first phase of the injection process, process $1 \rightarrow 2$ occurs at near constant volume $\left(V_{1 \rightarrow 2}=0.781 \mathrm{~cm}^{3}\right)$ and the second phase of the injection process, process $2 \rightarrow 3$ occurs at near constant pressure $(\approx 250 \mathrm{kPa})$ that results in the volume increase of the $\mathrm{CV}, V_{3}=1.212 \mathrm{~cm}^{3}$ (Fig. 33). Following the injection process $1 \rightarrow 2 \rightarrow 3$, the FPE expands isentropically during the process $3 \rightarrow 4$ to about $V_{4}=1.461 \mathrm{~cm}^{3}$, where the pressure is above atmosphere $\left(P_{4}=190 \mathrm{kPa}\right)$. Next, the blowdown phase of the exhaust process $4 \rightarrow 5$ occurs, where the expended working fluid is discharged due to the pressure gradient across the CV and exhaust system. Finally, the FPE undergoes the displacement phase of the exhaust process $5 \rightarrow 1$, where the residual working fluid is scavenged from the CV until the volume of the CV becomes $V_{1}=0.781 \mathrm{~cm}^{3}$. The ratio of the numerical integration of PV diagram (PdV work) and the enthalpy added to the FPE $\left(M_{3} c_{p} T_{i}-M_{1} c_{p} T_{i}\right)$ over one operating cycle gives the FPE energy conversion efficiency $\eta=13.78 \%$. Here, the injection ratio is $\mathrm{IR}=1.55$ and the expansion ratio is $\mathrm{ER}=1.2$. A corresponding plot of mass of the working fluid in the $\mathrm{CV}$ for an operating cycle is shown in Fig. $3 \mathrm{~b}$.

The peak internal cavity temperature across an operating cycle occurs at thermodynamic state 2, $T_{2}=403.7 \mathrm{~K}$ (Fig. 33), which is above the injection temperature $\left(T_{i}=373 \mathrm{~K}\right)$ - a result of the enthalpy addition to the cavity (CV). Therefore, the design temperature of the FPE should be above the working fluid injection temperature. In an operating cycle, the time durations and velocity amplitudes for 
the different processes are unequal (Fig. 3d). For instance, the forward motion of the piston $(1 \rightarrow 5)$ that comprises of multiple processes lasts for a time duration $t_{15}=17.6 \mathrm{~ms}$, while for the backward motion of the piston $(5 \rightarrow 1)$, lasts longer with a time duration $t_{51}=36.8 \mathrm{~ms}$. It is worth noting that this return motion can be governed by careful FPE design. For example, a motion controlled by an opposing piston expansion will adopt a faster return to TDC. This is discussed in greater detail in following sections.

\subsection{Effect of operating conditions}

The effect of operating conditions, namely working fluid injection temperature $T_{i}$, pressure $P_{i}$, and time duration $\bar{t}_{23}$ on the performance of the FPE characterized in terms of efficiency $\eta$ is presented in Fig. 4. Here, one operating condition $\left(e . g . T_{i}\right)$ is varied while the other two operating conditions $\left(P_{i}, \bar{t}_{23}\right)$ are held constant. For each set of operating conditions, the model Eqs. 1 15 are solved simultaneously to first generate a PV diagram, thereupon based on the PV diagram, the corresponding efficiency $\eta$, IR, and ER are computed.

The model Eqs. 115 predicts that for fixed $P_{i}$ and $\bar{t}_{23}$, an increase in $\bar{T}_{i}$ does not affect the efficiency $\eta$ (Fig. 4a). A closer look at the PdV work and enthalpy added shows that they remain constant for any $T_{i}$. Note that the efficiency which depends on IR and ER is also found to be constant. On the contrary, both the IR and ER increase with $P_{i}$ for fixed $T_{i}$ and $\bar{t}_{23}$, resulting in an increase in the efficiency $\eta$ (Figs. 4b, c). It is evident from the figure that the $\eta$ is more dependent on IR than ER. As the expander is "free-piston" type, the stroke length or BDC volume is not fixed, and varies based on the operating conditions. For instance, upon doubling the injection pressure $P_{i}$ from $175 \mathrm{kPa}$ to $351 \mathrm{kPa}$, the $\mathrm{BDC}$ volume $V_{5}$ increases from $1.12 \mathrm{~cm}^{3}$ to $1.87 \mathrm{~cm}^{3}$ (Fig. 4c) - a feature unique to free-piston based systems. For fixed $P_{i}$ and $T_{i}$, an increase in the $\bar{t}_{23}$ results in an initial increase in the efficiency $\eta$, which peaks at $\bar{t}_{23}=1.5$ and then drops by $5 \%$ and $10 \%$ for $150 \mathrm{kPa}$ and $250 \mathrm{kPa}$, respectively (Figs. 4 $\mathrm{d}$, e, f). As the injection time $\bar{t}_{23}$ decreases the injection process approaches a constant volume process (Fig. 4 ). Note that that as $\bar{t}_{23}$ reduces, IR also diminishes. As seen above, a reduced IR will result in lower efficiency. To maximize energy conversion efficiency $\eta$ of the FPE, we choose $\bar{t}_{23}=1.5$ for the subsequent analyses.

\subsection{Significant operating parameter}

Among the three operating parameters, only $P_{i}$ and $\bar{t}_{23}$ are found to dominate the performance of an FPE. To establish the most significant parameter among the two, a sensitivity analysis has been performed on the FPE outputs - efficiency $\eta$ and output power $\Omega$-by defining sensitivities $\frac{\delta \eta}{\delta \bar{P}_{i}}, \frac{\delta \eta}{\delta t_{23}}, \frac{\delta \Omega}{\delta \bar{P}_{i}}$ and $\frac{\delta \Omega}{\delta \bar{t}_{23}}$, where $\delta$ denotes partial difference operator. For the analysis, a parametric sweep is performed on the model Eqs. 1 [ 5 with FPE inputs $\bar{P}_{i}$ in the range $\epsilon$ $[1.5,3.5]$ and $t_{23} \in\{0.15,1.5,5,10\}$, and sensitivities are computed and plotted (Figs. 5a,b). Here, the output power is nondimensionalized with the scaling parameter $P_{o} V_{o} \omega$. For a specific condition of $\bar{P}_{i}$ and $\bar{t}_{23}$, if $\frac{\partial \eta}{\partial \bar{P}_{i}}>\frac{\partial \eta}{\partial \bar{t}_{23}}$, then the efficiency $\eta$ of the FPE is sensitive to injection pressure $\bar{P}_{i}$ compared to injection time duration $\bar{t}_{23}$ and vice versa. In our analysis, we observe that the data points (sensitivities) depicted by ' $\square$ ', ' $\circ$ ', ' $\triangle$ ', and '*' are above the line of equal sensitivity, implying the FPE performance parameters $\eta$ and $\Omega$ are more sensitive to $\bar{P}_{i}$ than $\bar{t}_{23}$ (Figs. 5a,b).

\subsection{Effect of physical parameters}

The FPE physical parameters, namely spring stiffness $k$, load $b$, and piston mass $m$ affect its efficiency $\eta$ and operating frequency $f$ (Fig. 6a-c). Here, one parameter $(e . g . k)$ is varied while the other two parameters $(m, b)$ are held constant.

An increase in spring stiffness $k$ restrains the piston motion causing shorter displacement strokes $\left(V_{2} \rightarrow\right.$ $\left.V_{4}\right)$, hence a lower $\mathrm{PdV}$ work and a decrease in the efficiency $\eta$. Also, the shorter displacement strokes reduce the piston-travel time, and hence an increase in the operating frequency $f$ (Fig. 6a). It is worth noting the significance of $k$ in this instance: this fundamentally represents the reset mechanism for the FPE. Careful design of $k$ can result in both the bestcase operating frequency and return to TDC functionality. Further, in a double-acting FPE design, 

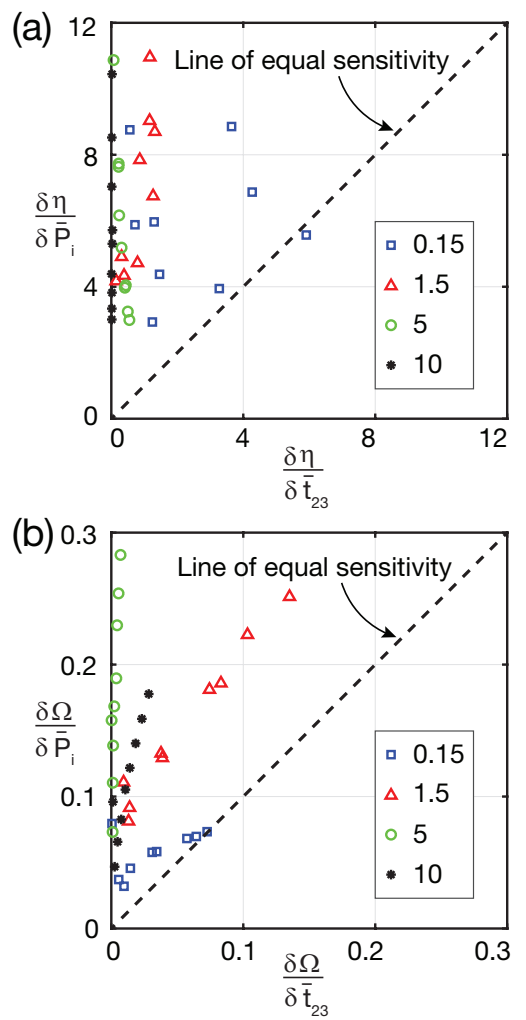

Figure 5: Sensitivity analysis on FPE performance, namely efficiency $\eta$ and nondimensional output power $\Omega$ on nondimensional injection pressure $\bar{P}_{i}$ and nondimensional injection time $\bar{t}_{23}$. For this, different injection pressures $\bar{P}_{i}$ in the range $\epsilon$ $[1.5,3.5]$ and injection times $\bar{t}_{23} \in\{0.15,1.5,5,10\}$ are used in the model. Each data point in the plot corresponds to a specific injection pressure $\bar{P}_{i}$.
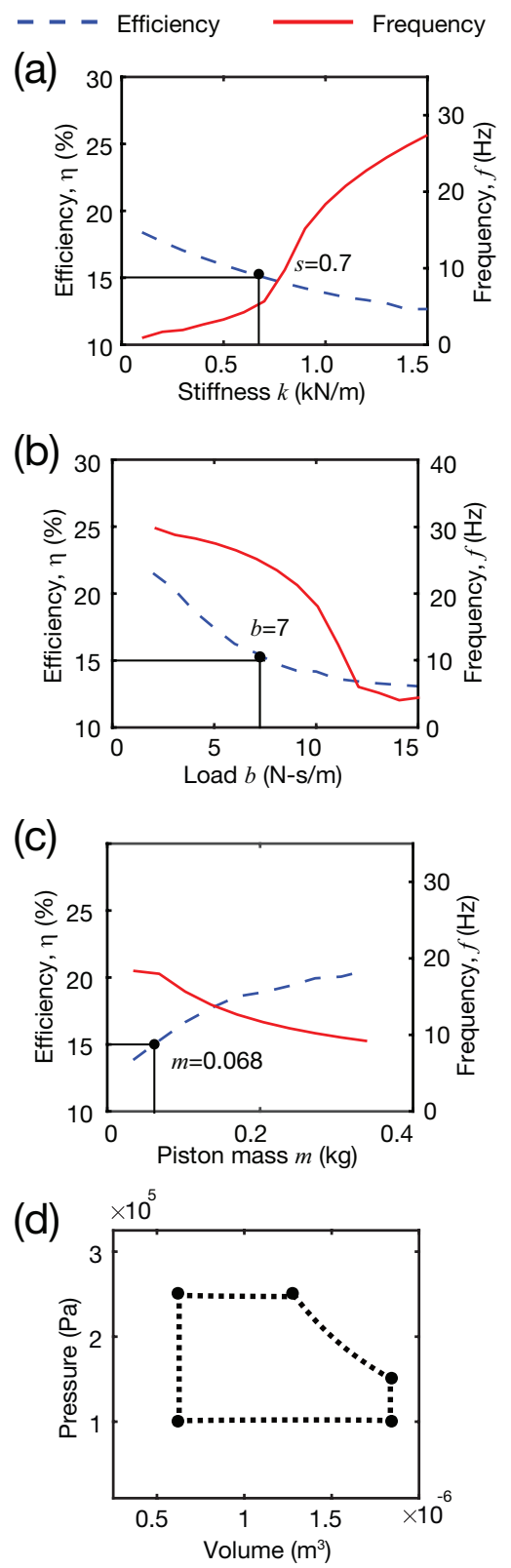

Figure 6: Effect of FPE physical parameters: spring stiffness $k$, load $b$, and piston mass $m$, on efficiency $\eta$ and frequency $f$ for the reference case with $P_{i}=250 \mathrm{kPa}, T_{i}=373 \mathrm{~K}$, and $\bar{t}_{23}=1.5$. (a) Fixed $b=10 \mathrm{~N}-\mathrm{s} / \mathrm{m}$ and $m=0.034 \mathrm{~kg}$ at different $k \in[100$, $1500] \mathrm{N} / \mathrm{m}$. (b) Fixed $k=1000 \mathrm{~N} / \mathrm{m}$ and $m=0.034 \mathrm{~kg}$ at different $b \in[2,15] \mathrm{N}-\mathrm{s} / \mathrm{m}$. (c) Fixed $b=10 \mathrm{~N}-\mathrm{s} / \mathrm{m}$ and $k=1000$ $\mathrm{N} / \mathrm{m}$ at different $m \in[0.034,0.34] \mathrm{kg}$. (d) PV diagram of the FPE with $k=700 \mathrm{~N} / \mathrm{m}, b=7 \mathrm{~N}-\mathrm{s} / \mathrm{m}$, and $m=0.068 \mathrm{~kg}$. 
the action of the opposing piston to limit the effective $k$ would be critical.

An increase in load $b$ resists the piston motion causing shorter displacement strokes $\left(V_{2} \rightarrow V_{4}\right)$, hence a lower $\mathrm{PdV}$ work and a decrease in the efficiency $\eta$. Despite the displacement stroke is shorter, the resistive behavior of the load $b$ slows the piston velocity resulting in longer cycle times or lower frequencies $f$ (Fig. 6p). It is worth noting, the ER and IR are found to decrease with the increase in $b$-consistent with efficiency trends.

Unlike stiffness $k$ and load $b$, an increase in piston mass $m$ results in increasing the efficiency due to higher IR and ER (Fig. 6.). For instance, $\mathrm{IR}=1.56$ and $\mathrm{ER}=1.2$ for $m=0.034 \mathrm{~kg}$, and $\mathrm{IR}=3.9$ and $\mathrm{ER}=1.5$ for $m=0.34 \mathrm{~kg}$. The higher $\mathrm{ER}$ and IR implies larger displacement strokes, and is a result of higher momentum gained by the piston during the the injection process $1 \rightarrow 3$. We acknowledge that the FPE operates close to its resonant frequency, and an increase in piston mass $m$ results in decreasing the frequency $f$ given by the relation $f=\sqrt{k / m}$.

To achieve an efficiency of at least $15 \%$, based on the physical parameters from Figs. 6a-c, an FPE with $k=0.7 \mathrm{kN} / \mathrm{m}, b=7 \mathrm{~N}-\mathrm{s} / \mathrm{m}$, and $m=0.068 \mathrm{~kg}$ is studied (Fig. 6d). The model predicts that the FPE generates a PdV work of $140 \mathrm{~mJ}$ at an efficiency of $18 \%$, which is operating at $16 \mathrm{~Hz}$ frequency. This corresponds to an output power of $2.24 \mathrm{~W}$. The achieved operating efficiency and output power places the FPE well above many comparably sized phasechange, low-temperature systems disclosed in literature [28].

\subsection{Effect of heat and pumping losses}

Using the FPE with parameters $k=0.7 \mathrm{kN} / \mathrm{m}, b=7$ $\mathrm{N}-\mathrm{s} / \mathrm{m}$, and $m=0.068$, the effect of heat loss and pumping loss are separately investigated over a range up to three orders of magnitude, $O\left(10^{3}\right)$ (Fig. 7) by choosing $\beta=0.0064 \mathrm{~kg} /(\mathrm{Pa}-\mathrm{s})$ and $h=0 \mathrm{~W} / \mathrm{K}$, respectively.

With the increase in $h$, the efficiency of the FPE decreases due to the increase in heat loss (Fig. 7 a). The heat loss coefficient $h$ used in this study is in the range of 0.00025 to $0.25 \mathrm{~W} / \mathrm{K}$, three orders of magnitude; the range of $h$ is based on our previous
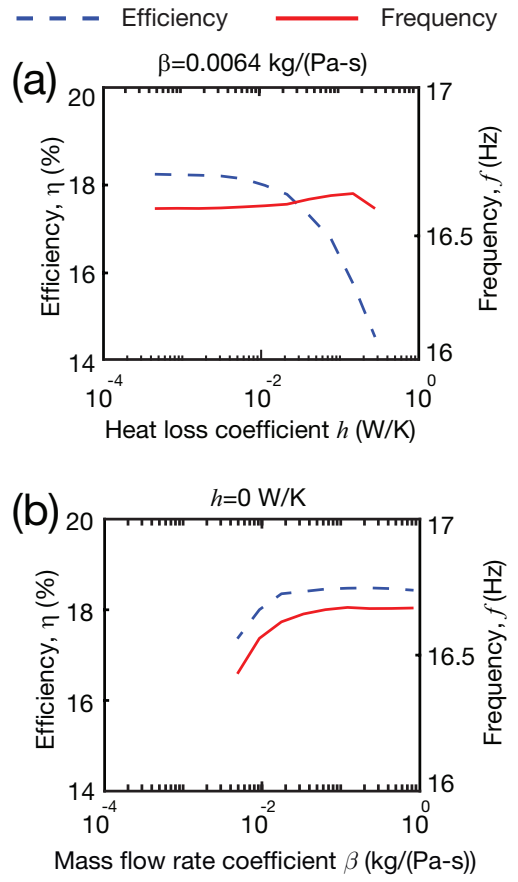

Figure 7: Effect of (a) heat loss coefficient $h$ and (b) mass flow rate coefficient $\beta$ on the efficiency of an FPE designed with physical parameters: piston mass $m=0.068 \mathrm{~kg}$, spring stiffness $k=700 \mathrm{~N} / \mathrm{m}$, and load $b=7 \mathrm{~N}-\mathrm{s} / \mathrm{m}$ operating with $P_{i}=250 \mathrm{kPa}$ $T_{i}=373 \mathrm{~K}$, and $\bar{t}_{23}=1.5$. 
work using model and experiment 29]. A heat loss coefficient $h, O\left(10^{-3}\right)$ results in a heat loss that is $O(\mathrm{PdV}$ work $)$. Therefore, for realizing a miniature FPE, the $h$ should be limited to $\sim 0.001 \mathrm{~W} / \mathrm{K}$, which results in an FPE efficiency of $18 \%$. Note that the heat loss has no significant effect on the frequency (Fig. 7a).

An increase in mass flow rate coefficient $\beta$ increases both the efficiency and frequency, because a higher $\beta$ implies a smaller resistance to flow or lower pumping loss (pumping work) during the exhaust process $5 \rightarrow 1$-resulting higher PdV work (Fig. 7b). The pumping loss is parasitic (friction-like) in nature, modeled as a mechanical damper, and exhibits the characteristics of load $b$. Therefore, an increase in $\beta$ that is decrease in load $b$ causes an increase in frequency - consistent with trends in Fig. 6b.

\section{Conclusions}

This work describes the behavior, performance and explores the design space of a centimeter-sized free piston expander, which is known to have several advantages over traditional turbine-style approaches. The FPE operates as an open cycle and is modeled using first principles. Three observations are reported that help achieve higher efficiencies: (1) a higher injection pressure; (2) an optimum nondimensionalized duration of injection time of $1.5 ;(3)$ softer springs, lower loads, heavier pistons are desirable. We identified the FPE's physical parameters, operating conditions, and acceptable heat loss and mass flow rate coefficients which yield an efficiency of $15 \%$ and an output work on the order of a few Watts. FPE performance reflects the influence of two critical parameters, injection pressure of the working fluid and duration of injection. These significantly effect output power and operating efficiency.

This study indicates the promise of the small-scale FPE approach for low temperature energy scavenging. The $15 \%$ operating efficiency is a significant improvement over many low temperature phase-change systems in literature. Further, the architecture of the FPE provides a more reliable, production ready system that can be achieved without the mechanical challenges faced by microscale turbine-based de-

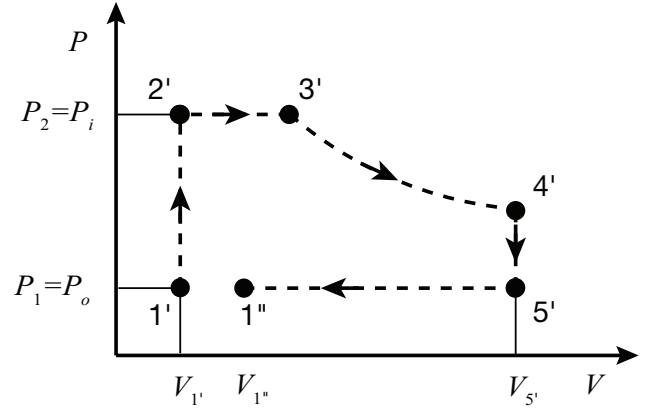

Figure A1: Transient pressure-volume diagram of the FPE in an open cycle operation. The superscript ' $"$ ' and ' $/$ ' denote transient states. Note that the cycle is not closed that is states $1^{\prime}$ and $1^{\prime \prime}$ are not equal. However, the FPE reaches a steady state operation (states $1^{\prime}$ and $1^{\prime \prime}$ are equal) in the subsequent cycles.

vices. This can be especially valuable when considering modern manufacturing processes like additive manufacturing.

\section{Appendix}

An example integration illustrating the first cycle in a transient dwell-up starting from state 1 is shown in Fig. A1. To generate a pressure-volume diagram, the model Eqs. $7 \sqrt{10}$ is numerically integrated in the order $1 \rightarrow 2 \rightarrow 3 \rightarrow 4 \rightarrow 5 \rightarrow 1$ starting with state 1 : $\Delta \bar{V}_{1}, \Delta \bar{P}_{1}, \Delta \bar{T}_{1}, \Delta \dot{\bar{V}}_{1}=0$ as described in the Model section. But, since the system undergoes a transient phase denoted with the superscript ' ', the cycle is not closed where $\Delta \bar{V}_{1}^{\prime} \neq \Delta \bar{V}_{1}^{\prime \prime}, \Delta \bar{P}_{1}^{\prime} \neq \Delta \bar{P}_{1}^{\prime \prime}$, and $\Delta \bar{T}_{1}^{\prime} \neq \Delta \bar{T}_{1}^{\prime \prime}$ (dashed line in Fig. A2). However, if the process is repeated, the cycle eventually reaches steady state, the cycle closes where $\Delta \bar{V}_{1}^{\prime}=\Delta \bar{V}_{1}^{\prime \prime}$, $\Delta \bar{P}_{1}^{\prime}=\Delta \bar{P}_{1}^{\prime \prime}$, and $\Delta \bar{T}_{1}^{\prime}=\Delta \bar{T}_{1}^{\prime \prime}$-and the steady states are denoted without superscript "' (solid line in Fig. A2). The integration stops when the equality has been satisfied to a specified tolerance. In practice, a function-minimization computational procedure was used, which determine the proper choice of $\Delta \bar{V}_{1}, \Delta \bar{P}_{1}, \Delta \bar{T}_{1}$ that would result in a steady-state periodic cycle. 


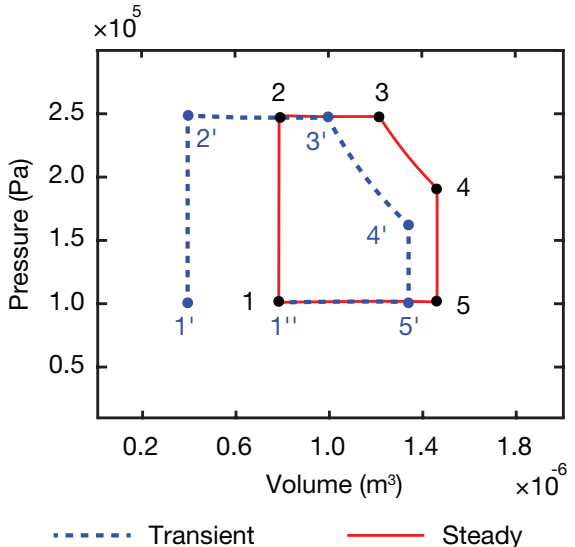

Figure A2: Transient and steady state pressure-volume diagrams of the FPE corresponding to the results presented in Fig. 32. Here, we deliberately start the FPE at an arbitrary piston location (state $1^{\prime}$ ) to demonstrate that the FPE reaches steady state (without the superscript ' ') in the next cycle - a result that our function-minimization computation procedure was able to predict (Fig. 3p).

\section{Acknowledgment}

This work was supported in part by Wichita State University capitalization funds and by the NSF via cooperative agreement OIA-1541079.

\section{Nomenclature}

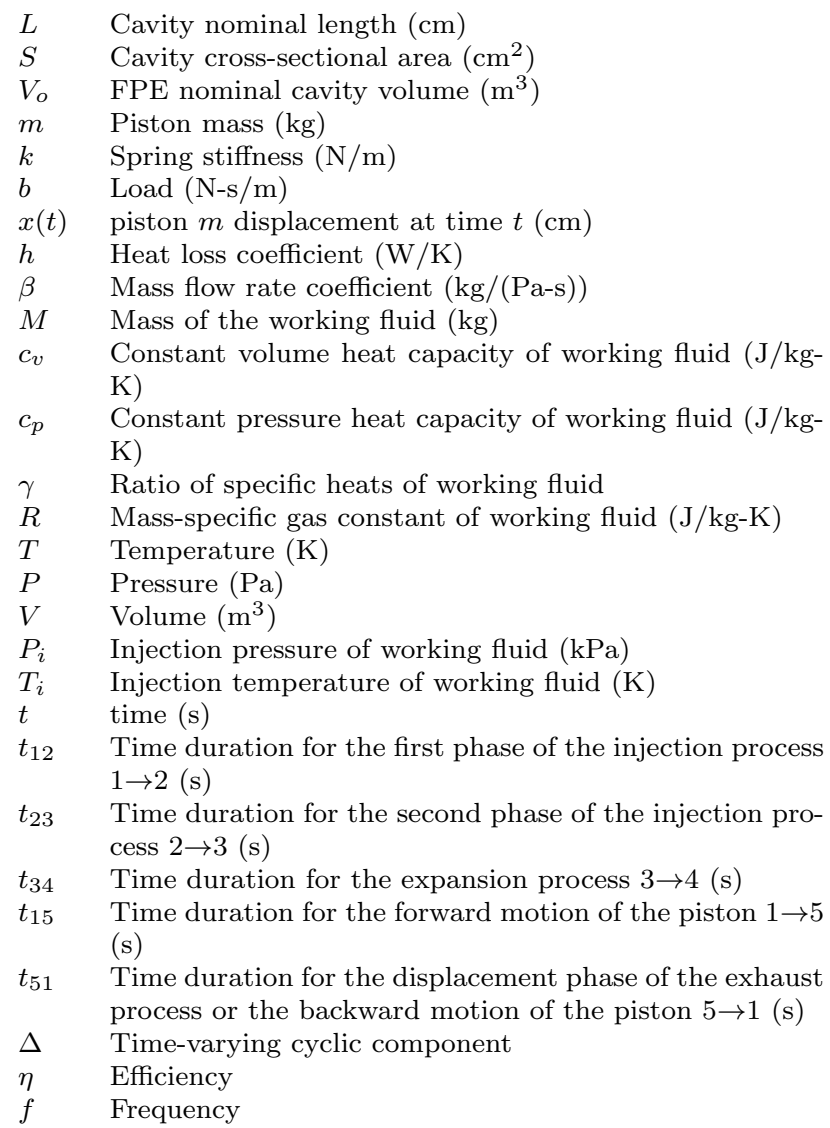

Abbreviations

FPE: Free piston expander

ORC: Organic Rankine cycle

HCCI: Homogeneous charge compression ignition

TDC: Top dead center

BDC: Bottom dead center

CV: Cavity or control volume

IR: Injection ratio

ER: Expansion ratio

Other Subscripts and Superscripts

Numerical subscript FPE's thermodynamic state

Overbar Nondimensional term

Overdot Differentiation with respect to time

Subscript $i$ Injection condition

Subscript o Ambient condition

Superscripts / and /" Transient condition 


\section{References}

[1] D. C. Walther, J. Ahn, Advances and challenges in the development of power-generation systems at small scales, Progress in Energy and Combustion Science 37 (5) (2011) 583-610.

[2] M. Imran, F. Haglind, M. Asim, J. Z. Alvi, Recent research trends in organic Rankine cycle technology: A bibliometric approach, Renewable and Sustainable Energy Reviews 81 (Part 1) (2018) $552-562$. doi:https://doi.org/10. 1016/j.rser.2017.08.028.

[3] C. S. III, C. Depcik, Review of organic rankine cycles for internal combustion engine exhaust waste heat recovery, Applied Thermal Engineering $51(1-2)(2013) 711-722$. doi:10.1016/j. applthermaleng.2012.10.017.

[4] M. Karvonen, R. Kapoor, A. Uusitalo, V. Ojanen, Technology competition in the internal combustion engine waste heat recovery: a patent landscape analysis, Journal of Cleaner Production 112 (2016) 3735-3743, 00016.

[5] Y. Xu, L. Tong, H. Zhang, X. Hou, F. Yang, F. Yu, Y. Yang, R. Liu, Y. Tian, T. Zhao, Experimental and simulation study of a free piston expanderlinear generator for small-scale organic rankine cycle, Energydoi:https: //doi.org/10.1016/j.energy.2018.07.171. URL http://www.sciencedirect.com/ science/article/pii/S0360544218314683

[6] T. Yamamoto, T. Furuhata, N. Arai, K. Mori, Design and testing of the organic rankine cycle, Energy 26 (2001) 239-251.

[7] B. Liu, K. Chien, C. Wang, Effect of working fluids on organic rankine cycle for waste heat recovery, Energy 29 (2004) 1207-1217.

[8] A. Giuffrida, A theoretical study on the performance of a scroll expander in an organic rankine cycle with hydrofluoroolefins (hfos) in place of r245fa, Energydoi:https: //doi.org/10.1016/j.energy.2018.07.146. 
URL http://www.sciencedirect.com/ science/article/pii/S0360544218314464

[9] B. Ziapour, Performance analysis of an enhanced thermosyphon rankine cycle using impulse turbine, Energy 34 (10) (2009) 1636 - 1641, 11th Conference on Process Integration, Modelling and Optimisation for Energy Saving and Pollution Reduction. doi:https: //doi.org/10.1016/j.energy.2009.07.012. URL http://www.sciencedirect.com/ science/article/pii/S0360544209002904

[10] G. Wenzhi, Z. Junmeng, L. Guanghua, B. Qiang, F. Liming, Performance evaluation and experiment system for waste heat recovery of diesel engine, Energy 55 (2013) 226 - 235. doi :https : //doi.org/10.1016/j.energy.2013.03.073. URL http://www.sciencedirect.com/ science/article/pii/S0360544213002703

[11] B. Lei, W. Wang, Y.-T. Wu, C.-F. Ma, J.-F. Wang, L. Zhang, C. Li, Y.-K. Zhao, R.-P. Zhi, Development and experimental study on a single screw expander integrated into an organic rankine cycle, Energy 116 (2016) $43-52$. doi:https: //doi.org/10.1016/j.energy.2016.09.089. URL http://www.sciencedirect.com/ science/article/pii/S0360544216313305

[12] L. C. Mendoza, J. Navarro-Esbr, J. C. Bruno, V. Lemort, A. Coronas, Characterization and modeling of a scroll expander with air and ammonia as working fluid Applied Thermal Engineering 70 (1) (2014) 630 $640 . \quad$ doi:https://doi.org/10.1016/j. applthermaleng.2014.05.069.

URL http://www.sciencedirect.com/ science/article/pii/S1359431114004451

[13] F. Formosa, L. G. Frchette, Scaling laws for free piston stirling engine design: Benefits and challenges of miniaturization, Energy 57 (2013) 796 - 808. doi:https: //doi.org/10.1016/j.energy.2013.05.009. URL http://www.sciencedirect.com/ science/article/pii/S0360544213003939
[14] X. Hou, H. Zhang, F. Yu, H. Liu, F. Yang, Y. Xu, Y. Tian, G. Li, Free piston expanderlinear generator used for organic Rankine cycle waste heat recovery system, Applied Energy 208 (Supplement C) (2017) 1297 - 1307. doi:10.1016/j.apenergy.2017.09.024.

[15] Y. Tian, H. Zhang, G. Li, X. Hou, F. Yu, F. Yang, Y. Yang, Y. Liu, Experimental study on free piston linear generator (FPLG) used for waste heat recovery of vehicle engine, Applied Thermal Engineering 127 (Supplement C) (2017) 184 - 193. doi:10.1016/j. applthermaleng.2017.08.031.

[16] G. Li, H. Zhang, F. Yang, S. Song, Y. Chang, F. Yu, J. Wang, B. Yao, Preliminary Development of a Free Piston Expander-Linear Generator for Small-Scale Organic Rankine Cycle (ORC) Waste Heat Recovery System, Energies 9 (4) (2016) 300.

[17] S. Qiu, L. Solomon, G. Rinker, Development of an integrated thermal energy storage and freepiston stirling generator for a concentrating solar power system, Energies 10 (9) (2017) 1361.

[18] C. Champagne, L. Weiss, Performance analysis of a miniature free piston expander for waste heat energy harvesting, Energy Conversion and Management 76 (2013) 883-892.

[19] S. Thapa, E. Borquist, L. Weiss, Thermal energy recovery via integrated small scale boiler and superheater, Energy 142 (2018) 765-772.

[20] S. Thapa, E. Borquist, A. Baniya, L. Weiss, Experimental and computational investigation of a mems-based boiler for waste heat recovery, Energy Conversion and Management 100 (2015) 403-413.

[21] B. Preetham, L. Weiss, Investigations of a new free piston expander engine cycle, Energy 106 (2016) 535 - 545. doi:10.1016/j.energy. 2016.03 .082 .

[22] S. Zhang, J. Wang, Z. Guo, Novel micro freepiston swing engine and its feasibility validation, 
Tsinghua Science and Technology 10 (3) (2005)

381 - 386. doi:10.1016/S1007-0214(05) 70085-7.

[23] H. T. Aichlmayr, D. B. Kittelson, M. R. Zachariah, Micro-hcci combustion: experimental characterization and development of a detailed chemical kinetic model with coupled piston motion, Combustion and Flame 135 (3) (2003) 227 - 248. doi:10.1016/S0010$2180(03) 00161-5$.

[24] B. S. Preetham, M. Anderson, C. Richards, Modeling of a resonant heat engine, Journal of Applied Physics 112 (12). doi:http://dx.doi. org/10.1063/1.4769447.

[25] R. Mikalsen, A. Roskilly, A review of freepiston engine history and applications, Applied Thermal Engineering 27 (14-15) (2007) 2339 2352. doi:10.1016/j.applthermaleng.2007. 03.015 .

[26] P. Achten, J. van den Oever, J. Potma, G. Vael, Horsepower with brains: The design of the chiron free piston engine, Society of Automotive Engineers (2000-01-2545).

[27] B. Preetham, M. Anderson, C. Richards, Estimation of parasitic losses in a proposed mesoscale resonant engine: Experiment and model, Journal of Applied Physics 115 (5) (2014) 054904 .

[28] L. Weiss, Power production from phase change in mems and micro devices, a review, International Journal of Thermal Sciences 50 (5) (2011) 639-647.

[29] H. Bardaweel, B. S. Preetham, R. Richards, C. Richards, M. Anderson, Mems-based resonant heat engine: scaling analysis, Microsystem Technologies 17 (8) (2011) 1251-1261. doi: 10.1007/s00542-011-1306-y

URL https://doi .org/10.1007/s00542-0111306-y 